Pathologe 2008 · [Suppl 2] 29:259-263

DOI 10.1007/s00292-008-1083-2

Online publiziert: 28. September 2008

(c) Springer Medizin Verlag 2008

\section{N. Grabe ${ }^{1,2}$}

${ }^{1}$ Institut für Medizinische Biometrie und Informatik, Sektion

Medizinische Informatik, Universitätsklinikum Heidelberg

2 Hamamatsu TIGA Center, BQ10, BIOQUANT Center, Heidelberg

\title{
Virtuelle Mikroskopie in der Systempathologie
}

Wesentliches Ziel der Systembiologie [1] ist die systematische Erfassung und Simulation möglichst großer und komplexer biologischer Zusammenhänge. Kernstück der Systembiologie ist dabei die Erschaffung von Modellen. Derartige Modelle besitzen gegenüber rein textuellen Beschreibungen von Systemen die Fähigkeit, Diagnosen über physiologische Systemzustände und physikalisch messbare Parameter in einen tatsächlichen direkten Bezug setzen zu können. Hiermit eröffnet die Systembiologie auch völlig neuartige Perspektiven für die Pathologie. Gegenwärtig führen die in der Pathologie eingesetzten Technologien und existierenden Spezialdisziplinen häufig zu fragmentierten Informationen. Dem Pathologen und dem behandelnden Arzt kommen hierbei die Aufgaben der Integration der verfügbaren Daten in ein ganzheitliches Krankheitsbild zu. Hieraus können wiederum Prognosen und Behandlungsoptionen für den einzelnen Patienten abgeleitet werden.

Diese Interpretation der qualitativen und quantitativen histologischen Messparameter erfolgt dabei generell in einem hochspezifischen klinischen und biologischen Kontext. An dieser Stelle ergänzen sich symbiotisch die Möglichkeiten der Systembiologie mit den Anforderungen an eine zukünftige Pathologie: Gerade die Modellierung von systembiologischen Zusammenhängen erlaubt es, diesen gesuchten Kontext für eine Diagnose und Prognose bereitzustellen. Durch die Verknüpfung systembiologischer Modellierung mit z. B. histologischen Auswertungsmöglichkeiten ergibt sich damit in
Zukunft die Möglichkeit der kausal begründeten Interpretation eines Gewebeschnittes durch im Modell enthaltene $\mathrm{Zu}$ sammenhänge. Grundlegend hierfür werden Technologien sein, die es erlauben, histologische Präparate im hohen Durchsatz vollautomatisch zu mikroskopieren. Dies ist die Domäne der virtuellen Mikroskopie.

Eine Reihe von Herstellern bietet inzwischen prinzipiell geeignete Geräte und Softwarelösungen an. Die ersten technischen Grundlagen für die Verbindung von Systempathologie und virtueller Mikroskopie sind daher heute bereits verfügbar. Die kommenden Jahre werden zeigen, wie hieraus weitergehende Ansätze für die Verbindung von virtueller Mikroskopie und Systempathologie entwickelt werden können. Die grundlegenden Punkte hierbei sind aus Sicht des Autors:
1. Pathologie hat sich in den letzten Dekaden immer weiter zu einem mechanistischen Verständnis von Erkrankungen entwickelt.

2. Genomics und Proteomics erlauben erstmals die umfassende Vermessung vollständiger molekularer Netzwerke.

3. Die Systembiologie erlaubt die Bildung molekularer und funktionaler In-silico-Modelle komplexer molekularer Systeme.

4. Die Systempathologie wird den Brückenschlag zwischen gestörten molekularen Netzwerken und einer krankhaften Gewebemorphologie ermöglichen (Genotyp-Phänotyp-Relation).

5. Die virtuelle Mikroskopie wird eine fundamentale morphologische Datenquelle für diese Brücke von molekularen Netzwerken zu Krankheitsmodellen darstellen.

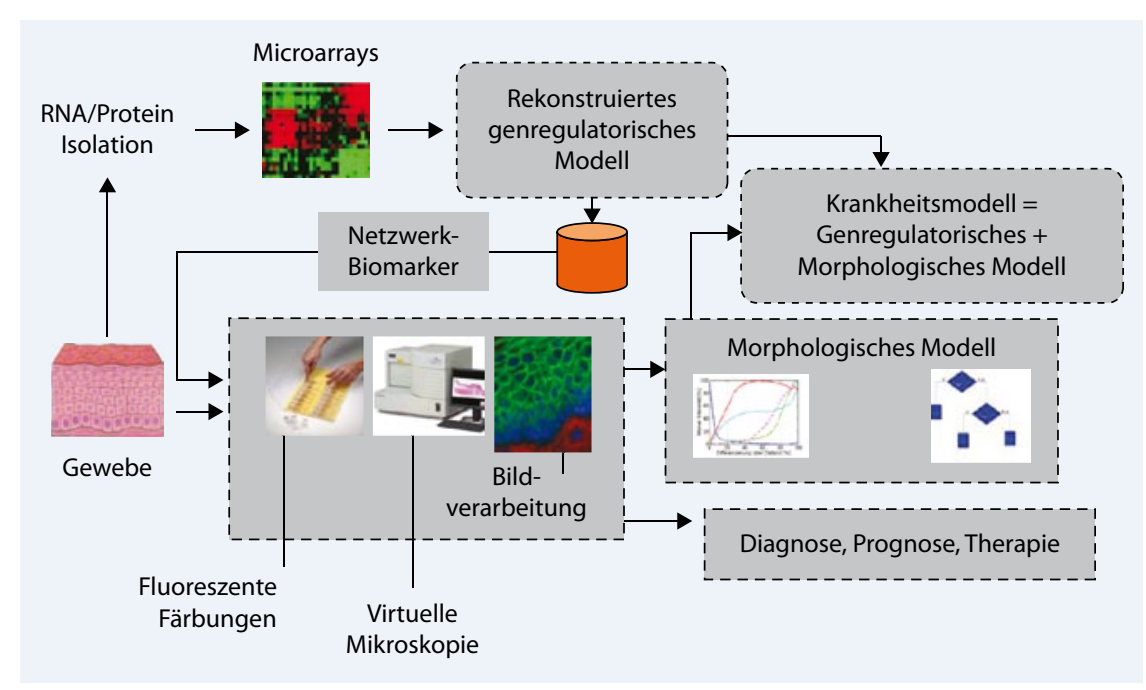

Abb. $1 \Delta$ Virtuelle Mikroskopie in der Systempathologie 
Hochdurchsatzanalysen als Wegbereiter der Systempathologie

Zunehmend spielt die quantitative Auswertung histologischer Präparate eine grundlegende Rolle innerhalb der Pathologie. Dies umfasst z. B. die grundlegende Quantifizierung zellulärer Eigenschaften und auch deren Verwertung innerhalb des diagnostischen Prozesses wie sie z. B. von Bartels gefordert wird [2]. Neben der histologischen Ebene spielt zunehmend die RNA-Expressionsebene eine zentrale Rolle. Mikroarrays erlauben die hochparallele Erhebung quantitativer Expressionsdaten. Durch diese starke Parallelisierung von Messungen können Hypothesen zu Krankheitsursachen, sprich fehlregulierte Gene, relativ leicht identifiziert werden. Problematisch ist hierbei grundsätzlich die qualitative Bewertung und Interpretation dieser Datenflut. Häufig wird hierzu der Ansatz des Clusterings verwendet [3]. Hierbei wird versucht, ähnlich verlaufende Expressionsprofile zueinander zu gruppieren. Aus derartigen Ähnlichkeiten kann nur in geringem Maße auf tatsächliche funktionale Zusammenhänge zwischen den Genen geschlossen werden. Daher kann eine derartige ClusterAnalyse noch nicht als Interpretation der Messergebnisse aufgefasst werden.

Etwas weitergehend ist der Ansatz, Genexpressionsignaturen zu verwenden. Derartige Signaturen sind Cluster, die die Zuordnung einzelner Patienten zu bestimmten Verlaufsformen von Erkrankungen (z. B. Tumorrezidiv) und damit implizit zu bestimmten Therapien erlauben. Generell besteht der Vorteil von Genexpressions-Clustern in der Vielzahl der von ihnen mit einbezogenen Gene. Es konnte gezeigt werden, dass z. B. bei Brustkrebs im Fall der neoadjuvanten Chemotherapie ein komplexer Prädiktor aus 74 Biomarkern im Gegensatz zu einzelnen Markern zu einer Patientenstratifikation in der Lage war [4]. Werden derartige Profile aus paraffiniertem Gewebe gewonnen, lassen sich Genexpressionsanalysen prinzipiell auch in Routineabläufe einbetten [5]. Der Vorteil von Genexpressionsprofilen ist dabei neben der Parallelität von Expressionsmessungen, dass Resultate aus kleinsten Nadelbiopsien gewonnen werden [6].
Unbeantwortet bleibt bei einem derartigen Vorgehen allerdings notwendigerweise die Frage nach dem tatsächlichen prädiktiven Wert. Insbesondere bei kleinsten Probenmengen ist die Gefahr, dass die Probe nicht aussagekräftig für den gesamten Gewebezustand ist, hoch: Es fehlt am histologischen Kontext. Ebenso ist die Aussagekraft oft rein statistischer Natur: Es fehlt oft an entsprechender funktionaler Aufklärung der gemessenen Parameter. Eine entsprechende molekularbiologische Rationale kann durch weitergehende Studien wie z. B. zur Regulation der entsprechenden Biomarker erfolgen. So konnte z. B. durch die Polymerasekettenreaktion (PCR) gezeigt werden, dass zuvor als potenziell prognostisch identifizierte Marker $\mathrm{HOXB13}_{13}$ IL17BR und $\mathrm{CH}-$ DH mit der Expression von ER, PR und HER2 bei Brustkrebs vermutlich korreliert sind [7].

Ein funktionales Verständnis für die genregulatorischen und histologischen Zusammenhänge der untersuchten Biomarker steigert den qualitativen Wert der identifizierten Biomarker daher erheblich. Entscheidend wird allerdings nicht nur eine weitere Anreicherung von Informationen auf der genetischen Ebene sein, sondern vielmehr eine Integration verschiedenster Information wie z. B. die von genetischen Signaturen mit ,single nucleotide polymorphisms“ (SNPs) mit immunhistochemischen und z. B. radiologischen Daten [7, 8]. Zusammenfassend lassen sich zwei wesentliche zukünftige Fragen für die Pathologie der Zukunft ableiten: 1. Wie kann eine systematische Modellierung der funktionalen Zusammenhänge der untersuchten Biomarker die Qualität von gemessenen Biomarker-Expressionsmustern erhöhen?

2. Wie kann die systematische räumliche Erfassung von Biomarker-Expressionsmustern den Entwurf von Krankheitsmodellen unterstützen?

\section{Systempathologie als nächster Schritt}

Eine sinnvolle Antwort auf beide Fragen stellt das Paradigma der Systempathologie dar. Die Systempathologie ergibt sich dabei durch die Symbiose der funktionalen Interpretation genetischer Expressions- daten mit der morphologischen Situation im Gewebe. Notwendiges Ziel der Systempathologie ist die Bildung integrierter molekularer und morphologischer Modelle von Störungen der normalen Gewebefunktion. Im Bereich der Toxikologie sind hierzu seit einiger Zeit erste Ansätze erkennbar. So wurde z. B. unter dem Begriff "phenotypic anchoring“ gezeigt, wie sich Genexpressionsprofile und morphologische Analysen ansatzweise miteinander verbinden lassen [9]. Morphologisch wurde dabei anhand von HE-Schnitten charakterisiert, wie sich der Uterus von mit einem Östrogenpuls behandelten Mäusen über eine Zeitskala von 72 Stunden strukturell reorganisiert. Parallel hierzu wurden Microarray-Daten von etwa 3500 Genen erhoben und geclustert. Die gleichzeitige Betrachtung von Genexpressionsprofilen und morphologischen Strukturänderungen des Gewebes wird dabei als "phenotypic anchoring“ bezeichnet und stellt somit eine beispielhafte Vorstufe der Systempathologie dar. Dieser Ansatz ist auch als Toxicogenomics zu finden [10].

Charakteristisch für die beiden genannten Ansätze ist, dass zwar Morphologie und Genetik parallel betrachtet werden, dies allerdings faktisch getrennt erfolgt und keine weitere Integration der genetischen mit den morphologischen Daten mehr erfolgt. Ziel der Systembiologie - und im übertragenen Sinne damit auch der Systempathologie - hingegen ist es, durch eine Modellierung komplexer biologischer Systeme mechanistische Vorstellungen von der Funktion eines Organismus oder eines Gewebes zu erlangen. Die Verknüpfung von morphologischer und genetischer Modellierung muss insofern ein Grundelement der Systempathologie sein.

\section{Der komplexe Prozess der Systempathologie}

Generell involvieren Projekte der Systempathologie notgedrungen eine Reihe verschiedener Disziplinen. Die Bedeutung der virtuellen Mikroskopie für die Systempathologie lässt sich gut darstellen, wenn letztere als Prozess wiedergegeben wird. Ein entsprechendes beispielhaftes Prozessschema auf abstrakter Ebene ist in - Abb. 1 dargestellt. Ausgehend von 
einem Gewebe können durch multiparametrische Analysen wie z. B. Microarrays Expressionsprofile im hohen Durchsatz erstellt werden. Es ergeben sich durch entsprechende Algorithmen z. B. genregulatorische Netzwerke direkt aus den Expressionsdaten (als Beispiel s. [11]).

Parallel zu den Expressionsstudien werden mithilfe von virtueller Mikroskopie Gewebedaten akquiriert. Hierbei können vollständige Gewebeschnitte oder auch Tissue-Microarrays [12, 13] eingesetzt werden. Mithilfe von Bildverarbeitung können anschließend systematisch quantitative Daten aus den digitalisierten Schnittbildern extrahiert werden. Ähnlich wie bei Mikroarrays können auch morphologische Parameter aus Zeitserien bzw. Verlaufsserien bestimmt werden. Dies erlaubt die systematische Rekonstruktion morphologischer Gewebeveränderungen. Durch entsprechende bioinformatische Algorithmen kann somit analog der Netzwerkrekonstruktion bei Microarrays ein dynamisches morphologisches Modell rekonstruiert werden. Die Integration des rekonstruierten genregulatorischen Modells aus den Mikroarray-Daten (auf mRNA- oder Proteinebene) mit dem rekonstruierten Modell der morphologischen Änderungen ergibt dann die gesuchte systempathologische Modellierung.

Es ist $\mathrm{zu}$ erwarten, dass derartige systempathologische Modelle in Zukunft die beiden eingangs gestellten Fragen für die Systempathologie als Pathologie der Zukunft werden beantworten können. Durch die erreichte integrierte Modellierung von Morphologie und genregulatorischer Ebene wird somit erreicht:

1. Die Qualität der Biomarker wird durch ihre Einbettung in das genregulatorische Modell der Krankheitsentstehung inhärent evaluiert.

2. Die Aussagekraft der Biomarker wird durch ihre Einbettung in ein räumliches Modell der zugrunde liegenden Gewebeveränderungen verstärkt.

Darüber stellt eine systempathologische Modellierung ein völlig neuartiges Werkzeug für die Analyse komplexer Erkrankungen dar. Durch entsprechende Simulationen werden Hypothesen in silico testbar, bevor aufwendige In-vitro- oder In-

Pathologe 2008 · [Suppl 2] 29:259-263 DOI 10.1007/s00292-008-1083-2

(c) Springer Medizin Verlag 2008

N. Grabe

Virtuelle Mikroskopie in der Systempathologie

\section{Zusammenfassung}

Genomics und Proteomics haben sich zur Systembiologie weiterentwickelt. Grundsätzliches Ziel ist hierbei die Erstellung komplexer funktionaler Modelle biologischer Systeme über molekulare Netzwerke. Derartige Modelle erlauben die Interpretation und Bewertung quantitativer Messergebnisse in einer neuen Qualität und einem erheblich vertieften funktionalen Verständnis. Im Unterschied zur reinen Systembiologie wird bei der Systempathologie die morphologische Gewebeebene gleichberechtigt neben der molekularen genregulatorischen Ebene stehen. Hierdurch kann die Systempathologie realistische Krankheitsmodelle entwerfen, die von direktem Nutzen für Diagnose, Prognose und Therapie sein werden. In der vorliegenden Arbeit wird ein generischer Prozess der Systempathologie entworfen, der ein morphologisches mit einem genregulatorischen Modell zu einem molekularen Krankheitsmodell integriert. Die fluoreszente virtuelle Mikroskopie wird hierbei von essenzieller Bedeutung sein. Sie liefert morphologische und molekulare Gewebedaten in hoher Auflösung und großem Durchsatz. Am Beispiel der epidermalen Differenzierung wird gezeigt, wie - mithilfe der virtuellen Mikroskopie - aus fluoreszenten Gewebeschnitten Proteinnetzwerke rekonstruiert werden können, die die räumlich-zeitliche Expression von Biomarkern quantitativ modellieren.

Schlüsselwörter

Systempathologie - Systembiologie - Virtuelle Mikroskopie · Differenzierung · Biomarker

\section{Virtual microscopy in systems pathology}

\section{Abstract}

Genomics and proteomics have evolved towards systems biology. The general goal here is the construction of complex, functional models of biological systems on the basis of molecular networks. Such models enable improved quality in interpretation and evaluation of quantitative measurements and afford a substantially deeper functional understanding. Systems pathology differs from systems biology by attaching the same importance to spatial modelling of tissue alterations as to gene regulatory modelling. In this way, systems pathology is able to deploy disease models for improved diagnosis, prognosis and therapy. In the present work a generic process for systems pathology is created, in- tegrating gene regulatory and morphological models towards molecular disease models. For this purpose, fluorescent virtual microscopy will be essential as it delivers morphological and molecular tissue data with high spatial resolution and high throughput. Using epidermal differentiation as an example, it is shown how - using virtual microscopy - the spatiotemporal expression of biomarkers can be modelled by reconstructing protein networks from fluorescent tissue sections.

Keywords

Systems pathology · Systems biology - Virtual microscopy · Differentiation - Biomarker 


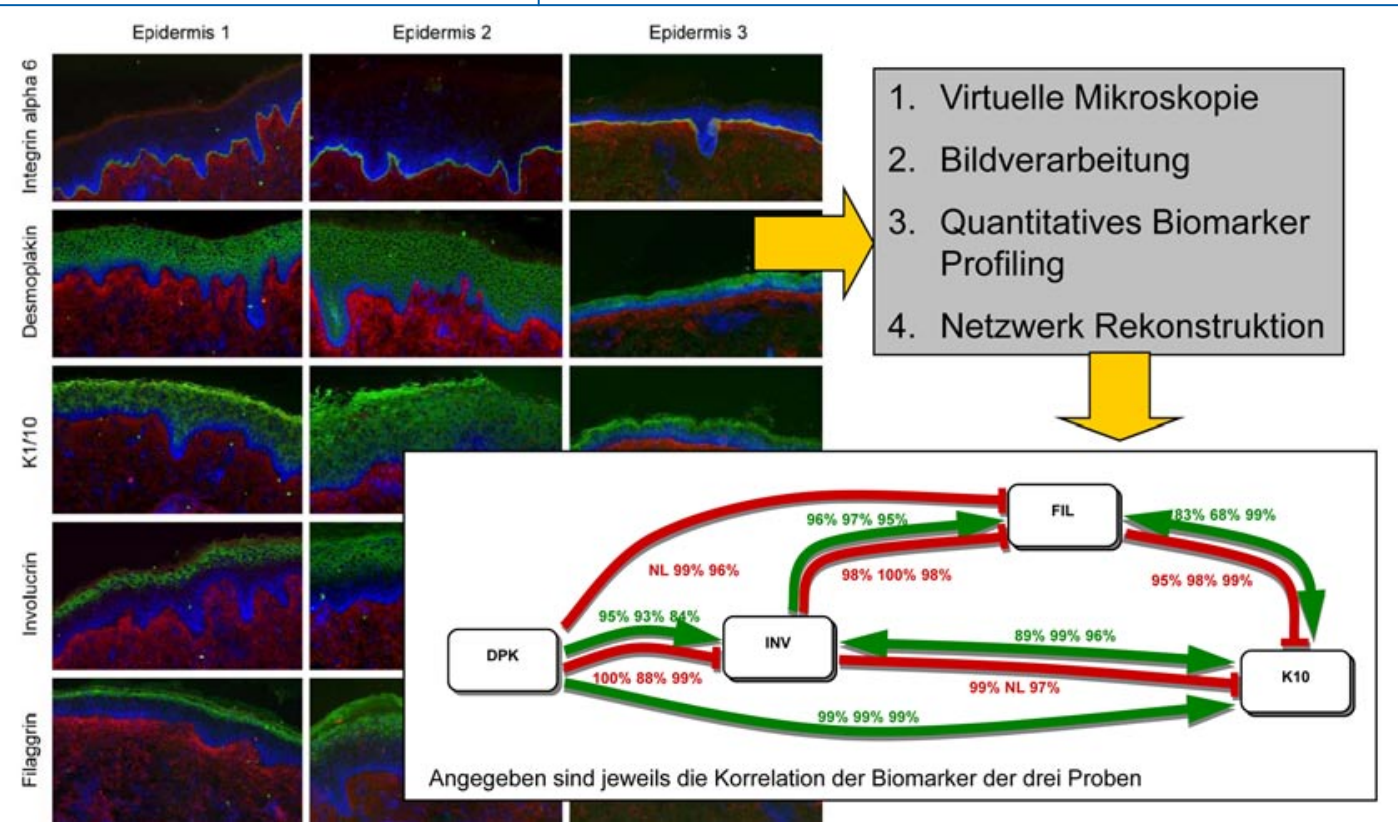

Abb. $2<$ Rekonstruktion molekularer Netzwerke der Keratinozytendifferenzierung
vivo-Studien erfolgen. Diese hier skizzierte „road-map“ der Systempathologie ist technisch herausfordernd, aber erreicht im Gegensatz zu den bisher dargestellten Ansätzen wie „phenotypic anchoring" eine tatsächliche Integration von räumlicher und genregulatorischer Modellierung.

\section{Virtuelle Mikroskopie}

Die virtuelle Mikroskopie erlaubt im hohen Durchsatz die automatisierte Mikroskopie von Objektträgern. Eine Reihe von Herstellern bietet entsprechende Geräte auf dem Markt an. Hierzu gehören Hamamatsu Photonics (Nanozoomer), Zeiss (Mirax), Aperio (Scanscope) und Olympus (Dot-Slide). Generell sind verschiedene Vergrößerungen von 20-63:1 möglich, wobei 40:1 derzeit aufgrund der Limitierung durch das Ölimmersionsobjektiv die für hohen Durchsatz maximal erreichbare Auflösungsgrenze darstellt. Die Mikroskopie erfolgt dabei im Durchlicht oder in der Fluoreszenz. Insbesondere in Kombination mit Tissue-Microarrays erlaubt die virtuelle Mikroskopie die systematische Gewinnung einer großen Zahl an Expressionsdaten.

Eines der bekanntesten Projekte hier$\mathrm{zu}$ ist der Human-Protein-Atlas (http:// www.proteinatlas.org), der eine systematische Charakterisierung aller humanen Gewebe und aller Proteine anstrebt [14]. Die derzeit im Human-Protein-Atlas er- hobenen Daten sind im Durchlichtmodus erhoben. Die Fähigkeit zur Fluoreszenz stellt allerdings für die Systempathologie eine grundlegende Anforderung dar. Fluoreszenz erlaubt die Trennung verschiedener Signale in eigenen Farbkanälen. Durch die Verwendung entsprechender Filter können auch räumlich überlagerte Farbsignale eindeutig voneinander getrennt und auch quantifiziert werden. Beispielhaft seien hier Doppelimmunfluoreszenzen mit einer zusätzlichen Kernfärbung zu nennen. Durch Fluoreszenz können die für eine morphologische Analyse notwendigen Messdaten, also die räumliche mRNA- oder Proteinexpression, quantitativ aus den untersuchten Geweben gewonnen werden, sodass sie für eine systembiologische räumliche Modellierung verwendet werden können.

\section{Rekonstruktion von Proteinnetz- werken aus Gewebeschnitten}

Es ist wichtig, an dieser Stelle darzustellen, dass Systempathologie ein wissenschaftliches Paradigma darstellt, welches eine Reihe von Jahren für erste Resultate benötigen wird. Eine ganze Reihe einzelner Bausteine dieses Paradigmas sind Gegenstand aktueller Forschungstätigkeit. Dies war die Kernmotivation für die Gründung des „Hamamatsu Tissue Imaging and Analysis“ (TIGA; http://www.tiga. uni-hd.de) an der Universität Heidelberg als Gemeinschaftsprojekt des Instituts für
Pathologie und des Instituts für Medizinische Biometrie und Informatik. Das TIGA ist im Rahmen des systembiologischen Heidelberger BIOQUANT Zentrums etabliert und an die Gewebebank des Heidelberger NCT (Nationales Zentrum für Tumorerkrankungen) angebunden (s. Beitrag in dieser Ausgabe von Herpel, Koleganova und Schirmacher).

Die derzeitigen Arbeiten zielen auf die Erarbeitung von Grundlagen für medizinische Anwendungen der Systembiologie wie z. B. in der Systempathologie. Hierzu gehören Modellierungsansätze von Geweben $[15,16]$ wie auch pathologischer Gewebezustände [17]. Vergleichbare Arbeiten laufen an einer Reihe anderer Forschungseinrichtungen $[18,19,20,21]$.

Beispielhaft soll hier ein am TIGA entwickeltes integriertes Verfahren zur systematischen Rekonstruktion morphologischer Netzwerke aus histologischen Schnittbildern dargestellt werden [22]. Zielstellung war die quantitative Vermessung der Differenzierung epidermaler Keratinozyten. Hierzu werden Gewebeschnitte der menschlichen Haut jeweils mit einer fluoreszenten Doppelimmunfärbung und einer zusätzlichen Kernfärbung gefärbt. Die Doppelimmunfärbung stellt dabei sowohl einen morphologischen Referenzmarker (Kollagen I) und einen Biomarker dar. Als typische Referenzmarker der epidermalen Differenzierung wurden dabei Filaggrin, Involucrin, Desmoplakin, 
Keratin K1/10 und Integrin alpha 6 untersucht (• Abb. 2).

Auf jedem Gewebeschnitt wurde dabei ein anderer Biomarker zusammen mit Kollagen 1 angefärbt. Die gewählten Biomarker repräsentieren verschiedene epidermale Schichten. Während Integrin alpha 6 an der Grenze zwischen Epidermis und Dermis exprimiert wird, ist Desmoplakin schwach bereits innerhalb der ersten Zelllage exprimiert. In höheren Schichten erfolgt dann eine Verstärkung der Expression parallel zu Keratin K1/10. Involucrin und Filaggrin gelten als jeweils frühe und späte Marker der epidermalen Differenzierung.

Es stellte sich die Frage, wie ein derartiges Expressionsprofil räumlich aus Schnittbildern extrahiert werden kann. Alle fluoreszenten Schnittbilder wurden mithilfe der virtuellen Mikroskopie digitalisiert. Mithilfe von Bildverarbeitung konnten entsprechende quantitative räumliche Expressionsprofile für die einzelnen Biomarker bestimmt werden (- Abb. 2). Aus derartigen Profilen können nun - analog zu Genexpressionsdaten aus Microarrays - temporale Proteinnetzwerke rekonstruiert werden. Zwei parallel aufsteigende Flanken weisen dabei potenziell auf eine fördernde Koregulation hin, während 2 parallel absteigende Flanken innerhalb der Profile auf eine hemmende Koregulation hindeuten. Durch Bestimmung des Korrelationskoeffizienten kann die Stärke der potenziellen Koregulation quantifiziert werden. Die prozentuale Zeitdauer, über die die Flanken parallel verlaufen, gibt dabei Auskunft über die Konfidenz der Parallelität. Ausgehend von diesen Überlegungen wurde ein entsprechendes Proteinnetzwerk rekonstruiert, das den temporalen Verlauf der zentralen Parameter der epidermalen Homöostase wiedergeben kann. Hierbei geben grüne Pfeile fördernde Koregulationen und rote hemmende Koregulationen an. Mit diesem Ansatz wurde ein erstes Beispiel für eine quantitative Rekonstruktion der epidermalen Homöostase auf räumlicher und Proteinexpressionsebene erreicht.

\section{Fazit für die Praxis}

Die Pathologie hat sich in den letzten Dekaden immer weiter hin zu einem me- chanistischen Verständnis von Erkrankungen entwickelt. Eine wesentliche Rolle spielen hierbei Genomics and Proteomics, die sich inzwischen zur Systembiologie weiterentwickeln. Bezogen auf die Pathologie ist daher in Zukunft die Entstehung der Systempathologie bzw. der medizinischen Systembiologie absehbar. Wesentlicher Unterschied zwischen Systembiologie und Systempathologie ist dabei die Integration von räumlichen Modellen von Geweben mit den entsprechenden genregulatorischen Modellen. Dadurch wird die virtuelle Mikroskopie als Datenlieferant für derartige Modelle unverzichtbar. Insbesondere gilt es, die virtuelle Mikroskopie mit einer anschließenden Bildverarbeitung zu einer räumlichen Modellbildung von Geweben weiterzuentwickeln. Ein solches Beispiel mit dem Ziel der Modellierung der epidermalen Homöostase wurde gezeigt.

\section{Korrespondenzadresse}

\section{N. Grabe}

Institut für Medizinische Biometrie und Informatik

Sektion Medizinische Informatik

Universitätsklinikum Heidelberg

Im Neuenheimer Feld 305, 69120 Heidelberg

niels.grabe@med.uni-heidelberg.de

Interessenkonflikt. Keine Angaben.

\section{Literatur}

1. Kitano H (2002) Computational systems biology. Nature 420: 206-210

2. Bartels PH (2000) Future directions in quantitative pathology: digital knowledge in diagnostic pathology. J Clin Pathol 53: 31-37

3. Fertuck KC, Eckel JE, Gennings C et al. (2003) Identification of temporal patterns of gene expression in the uteri of immature, ovariectomized mice following exposure to ethynylestradiol. Physiol Genomics 15: 127-141

4. Ayers M, Symmans WF, Stec J et al. (2004) Gene expression profiles predict complete pathologic response to neoadjuvant paclitaxel and fluorouracil, doxorubicin, and cyclophosphamide chemotherapy in breast cancer. J Clin Oncol 22: 2284-2293

5. Gianni L, Zambetti M, Clark K et al. (2005) Gene expression profiles in paraffin-embedded core biopsy tissue predict response to chemotherapy in women with locally advanced breast cancer. J Clin Oncol 23: 7265-7277

6. Pusztai L, Ayers M, Stec J et al. (2003) Gene expression profiles obtained from fine-needle aspirations of breast cancer reliably identify routine prognostic markers and reveal large-scale molecular differences between estrogen-negative and estrogen-positive tumors. Clin Cancer Res 9: 2406-2415
7. Bentzen SM (2008) From cellular to high-throughput predictive assays in radiation oncology: challenges and opportunities. Semin Radiat Oncol 18: 75-88

8. Costa J (2008) Is clinical systems pathology the future of pathology? Arch Pathol Lab Med 132(5): 774-776

9. Moggs JG, Tinwell H, Spurway T et al. (2004) Phenotypic anchoring of gene expression changes during estrogen-induced uterine growth. Environ Health Perspect 112: 1589-1606

10. Waters MD, Fostel JM (2004) Toxicogenomics and systems toxicology: aims and prospects. Nat Rev Genet 5: 936-948

11. Busch H, Camacho-Trullio D, Rogon Z et al. (2008) Gene network dynamics controlling keratinocyte migration. Mol Syst Biol 4: 199

12. Sauter G, Mirlacher M (2002) Tissue microarrays for predictive molecular pathology. J Clin Pathol 55: 575-576

13. Skacel M, Skilton B, Pettay JD et al. (2002) Tissue microarrays: a powerful tool for high-throughput analysis of clinical specimens: a review of the method with validation data. Appl Immunohistochem Mol Morphol 10: 1-6

14. Uhlen M, Bjorling E, Agaton C et al. (2005) A human protein atlas for normal and cancer tissues based on antibody proteomics. Mol Cell Proteomics 4: 1920-1932

15. Grabe N, Neuber K (2005) A multicellular systems biology model predicts epidermal morphology, kinetics and Ca2+ flow. Bioinformatics 21: 35413547

16. Grabe N, Pommerencke T, Muller D et al. (2006) Modelling epidermal homeostasis as an approach for clinical bioinformatics. Stud Health Technol Inform 124: 105-110

17. Grabe N, Neuber K (2007) Simulating psoriasis by altering transit amplifying cells. Bioinformatics 23: 1309-1312

18. Meineke FA, Potten CS, Loeffler M (2001) Cell migration and organization in the intestinal crypt using a lattice free model. Cell Proliferation 34: 253-266

19. Morel D, Marcelpoil R, Brugal G (2001) A proliferation control network model: the simulation of twodimensional epithelial homeostasis. Acta Biotheor 49: 219-234

20. Noble D (2004) Modeling the heart. Physiology (Bethesda) 19: 191-197

21. Walker D, Southgate J, Hill G et al. (2004) The Epitheliome: agent based modelling of the social behaviour of cells. BioSystems 76: 89-100

22. Grabe N, Pommerencke T, Steinberg T et al. (2007) Reconstructing protein networks of epithelial differentiation from histological sections. Bioinformatics 23: $3200-3208$ 